ECONOMÍA: TEORÍA Y PRÁCTICA • Nueva Época, número 44, enero-junio 2016, pp. 193-215, http://www.izt.uam.mx/economiatyp/ojs

\title{
Definición del mercado de trigo cristalino en el valle del Yaqui, México*
}

\section{Hard Wheat Market Definition in Yaqui Valley, Mexico}

\author{
María Cristina Garza Lagler** y Cristina Taddei Bringas***
}

\section{RESUMEN}

El valle del Yaqui es una región productora de trigo en el noroeste de México, cuyas agroempresas tienen una participación creciente en el mercado internacional. El objetivo de este trabajo es una aproximación a la definición del mercado del trigo cristalino que se produce en su territorio, para lo cual se realizó un análisis descriptivo apoyado en entrevistas a actores estratégicos del sistema de producción. Los resultados indican que este grano tiene tres mercados: la industria de alimentos balanceados para ganado, la industria procesadora de pastas y galletas en México y la exportación a países del norte de África, Sudamérica e Italia, principalmente. Cada uno se define por las condiciones que impone y por el tipo de producto que demanda.

Clasificación JEL: D21, D43, M210, Q110.

Palabras clave: mercado, estrategia, calidad, demanda, producción, comercialización, variedad, calidad.

\begin{abstract}
The Yaqui Valley is a wheat-producing region in northwestern Mexico, whose agribusinesses have a growing participation in the international market. The aim of this paper is an approach to a market definition of durum wheat produced in this region, which descriptive analysis is supported by interviews with strategic stakeholders in the wheat production system. The results indicate that the durum wheat in the Yaqui Valley has three markets: the feed industry, the processing industry for biscuits in Mexico and export to countries in North Africa, South America and Italy, mainly. Each is defined by the conditions imposed and the type of product they require.
\end{abstract}

JEL classification: D21, D43, M210, Q110.

Keywords: market, strategy, quality, demand, production, marketing, variety, quality.

\footnotetext{
* Fecha de recepción: 30/01/14. Fecha de aprobación: 20/01/2016.

** Centro de Investigación en Alimentación y Desarrollo, AC. Correo electrónico: mcristina. garza@gmail.com.

*** Coordinación de Desarrollo Regional del Centro de Investigación en Alimentación y Desarrollo, AC. Correo electrónico: ctaddei@ciad.mx.
} 


\section{INTRODUCCIÓN}

Los cambios en la demanda de los consumidores de alimentos y el incremento de sus exigencias se ven reflejados en transformaciones productivas (Klerkx y Leeuwis., 2008) y organizativas (Robles y Garza, 2010), así como en nuevos requerimientos de investigación y desarrollo (Smits, 2002). Esta transición invita a los mercados agrícolas a comportarse como estructuras oligopólicas en las que las estrategias juegan un papel relevante para las firmas, ya que les permiten no sólo posicionarse, sino, además, imponer barreras a sus competidores.

Los sistemas alimentarios son cada vez más complejos, debido a que incorporan diversos elementos, tales como los avances en la investigación, al tiempo que adoptan una serie de iniciativas, como alianzas, adquisiciones, fusiones, empresas conjuntas, asociaciones y acuerdos menos formales (Suárez y Bejarano, 2001). Es decir, pasan de una producción masificada, cuyos requerimientos tecnológicos y de conocimiento tenían un carácter homogéneo, a una de productos, procesos y organización diversificada y flexible, en constante actualización y en contacto con la demanda, para anticipar las exigencias de los consumidores. En tal sentido, es de esperarse que para los sistemas alimentarios se perfilen esquemas oligopólicos en los que las fuentes de conocimiento son fundamentales para el control y la concentración del mercado.

El valle del Yaqui, ubicado en la región noroeste de México, se caracteriza por su producción agrícola de altos rendimientos y de gran aceptación en los mercados internacionales. Este valle ha sido materia de disputa desde principios del siglo pasado entre la clase política y los grupos indígenas de la región (yaquis). También ha sido incubadora de desarrollos científicos importantes en agricultura y alimentos, por ejemplo, el realizado por Norman Borlaug, laureado con el Nobel de la Paz en 1970 y conocido como el padre de la revolución verde.

Actualmente, la relación investigador-productor se traduce en una mejora constante en las variedades vegetales, principalmente de trigo, resistentes a las condiciones imperantes en la región y que alcanzan muy altos rendimientos. Esto le da al agricultor la seguridad de sembrar una semilla fuerte que le permite insertarse en su mercado objetivo.

Al respecto, la pregunta que se plantea es: ¿cuál es ese mercado? Seguida de: ¿para qué sirve conocer el mercado? Dar respuestas a ellas requiere, antes que nada, definir qué es un mercado. En el caso del mercado del trigo cristalino en el cual convergen agroempresas del valle del Yaqui y firmas compradoras de este grano, es necesario identificar sus límites como primer paso 
para el análisis económico que interesa a los tomadores de decisiones. Así, el objetivo de este artículo es una aproximación a la definición del mercado de trigo del valle del Yaqui.

La estrategia metodológica seguida para ello tiene dos componentes: uno lo constituye la revisión de literatura en materia de desarrollos teóricos que aborden los factores históricos y económicos la región y de la producción de trigo. El segundo es la realización de entrevistas a los principales actores del sistema productivo de trigo cristalino del valle, para detectar los elementos de dicho sistema: agroempresas (oferentes), mercado (demandantes), investigación y desarrollo (ID), gobierno, y oferta de semilla para siembra del sector público y de la iniciativa privada. Posteriormente, se entrevistó a actores específicos de estos aspectos. Estas entrevistas fueron analizadas con las técnicas de análisis del discurso, siguiendo los lineamientos de la teoría fundamentada -metodología usada en análisis de carácter cualitativo; véase Glaser y Strauss (1999)-, para lo cual se utilizó el software MaxQda, versión $11^{1}$.

El trabajo se integra por cuatro secciones. En la primera se definen conceptos para el análisis procedentes de la teoría de la organización industrial, campo de la economía que estudia el comportamiento de los mercados. La segunda parte describe las características de la región estudiada, mientras que en la tercera se analizan la dimensión y estructura del mercado de trigo cristalino. En la última sección se resumen los principales resultados de la investigación.

\section{FUNDAMENTO TEÓRICO}

Hablar de mercado, en ciencia económica, es hablar de su funcionamiento, por lo que gran parte de los estudiosos de este campo se enfocan en el análisis de las fuerzas que interactúan en éste. Desde la perspectiva de la organización in-

\footnotetext{
${ }^{1}$ Durante una estancia en el Campo Experimental Noreste del Instituto Nacional de Investigación Forestal, Agraria y Pecuaria (INIFAP), de enero a marzo de 2013, se entrevistó sobre la ID al encargado del área de gestión y transferencia de tecnología de esa institución. En el caso del componente gubernamental, se acudió a la entonces presidenta del distrito de desarrollo rural. En lo que respecta a la oferta de semilla para siembra se entrevistó a los directivos de los dos únicos organismos de la región, uno privado y otro público. Sobre la oferta de grano, se entrevistó al gerente general de la agroempresa que agrupa al mayor número de productores de la región, cuyos volúmenes de comercialización son los más elevados del país. Finalmente, respecto a la demanda de grano, se acudió con el gerente de comercialización de la empresa más representativa de producción de sémola en México.
} 
dustrial, rama especializada en el estudio de los mercados, más allá de su función como espacio donde compradores y vendedores intercambian mercancías, un rasgo esencial de los mercados es que estén constituidos por bienes mutuamente sustituibles (Shepherd y Shepherd, 2003). La importancia de esta definición radica en el hecho de que sólo hasta que se determina su alcance real se puede comenzar a analizar la competencia en el mercado, es decir, una vez que se logra delimitar sus fronteras, identificando los bienes sustitutos y excluyendo los que no lo son.

Sin embargo, la delimitación de un mercado es un proceso complejo que implica estudiar las condiciones que impone la demanda, es decir, la zona de decisión del consumidor respecto a un producto, lo cual Shepherd y Shepherd $(2003)^{2}$ estiman a partir de lo siguiente:

- Elasticidad cruzada de la demanda: mide la sensibilidad en la demanda de un bien ante cambios en el precio de otro, de tal manera que si ante un aumento en el precio del bien $Y$, se incrementa la demanda del bien $X$, esto indicaría que ambos serán sustitutos y que se encuentran en un mismo mercado. En la práctica, hacer uso de las elasticidades resultaría complicado y muy costoso.

- Tipo de producto: análisis del uso y las características de los bienes o, en ciertos casos, de los reportes financieros anuales de las firmas.

- Dimensiones del producto: a partir de los precios, ya que sus variaciones pueden explicar qué tan sustituibles son o no los bienes.

- Área geográfica: en términos de mercado, más allá de la identificación de una zona, un buen indicador es el costo del transporte en relación al valor de los bienes. ${ }^{3}$ Otros dos indicadores son la distancia y el esquema de envío. La primera indica el radio del mercado del bien, mientras que el segundo determinará qué tan extenso es.

\footnotetext{
${ }^{2}$ Estos autores definen el mercado como un grupo de compradores y vendedores que intercambian bienes altamente sustituibles. Esta característica es fundamental para definir el mercado, ya que, por ejemplo, si un consumidor desea adquirir un televisor, tendrá que elegir entre los diversos televisores existentes y no entre bienes cuyas características no los hacen sustitutos, como un automóvil o una lavadora. Éstos, al no ser sustituibles unos por otros, por tanto, pertenecen a mercados distintos.

${ }^{3}$ En este punto, Shepherd y Shepherd (2003) usan como ejemplo los ladrillos. Éstos tienen un elevado costo de traslado en relación con su valor, de tal forma que su mercado está muy limitado geográficamente. El caso contrario sería el de los diamantes.
} 
Por otro lado, definir el mercado a partir de herramientas como la elasticidad cruzada de la oferta, que permite conocer los cambios en el bien ofrecido ante cambios en el precio de otro, puede resultar igualmente complejo. Baker (2007) realiza un análisis sobre la definición del mercado a partir de algunos casos judiciales en las cortes de Estados Unidos contra empresas acusadas de prácticas monopólicas. Este autor indica que en estas circunstancias, el análisis de la oferta llevaría a definir el mercado a partir de las fusiones de las empresas que participan en él y que, por tanto, la elasticidad de la oferta puede sesgarse, por lo que es necesario recurrir a otras herramientas, como identificar a los participantes del mercado, estudiar los efectos competitivos o evaluar las condiciones para el ingreso.

Entonces, definir un mercado desde la oferta es conocer su estructura (el contenido), la cual tiene que ver principalmente con la distribución por tamaño de las firmas competidoras (Taddei, 2008). Por su parte, Shepherd y Shepherd (2003) proporcionan los tres elementos de análisis que determinan el tipo de estructura de mercado en que se compite, a saber:

a) Cuota de mercado. Por su participación en el mercado, como porcentaje de las ventas totales de determinada industria o sector, la firma más grande que concurre puede ser dominante, mediana o pequeña. Este indicador por sí mismo reviste una gran importancia para definir si existe monopolio.

b) Concentración y número de rivales comparables. El número de rivales importantes puede ser decisivo, no así la cantidad total de firmas competidoras en el mercado, en la cual pueden incluirse algunas pequeñas. La concentración se considera una medida que hace evidente el grado de oligopolio.

c) Condiciones para el ingreso. Éstas pueden ser barreras que afecten la capacidad de competidores potenciales que se hallan fuera del mercado para entrar y convertirse en competidores de hecho. Cualquier cosa que disminuya la probabilidad, el alcance o la velocidad de acceso de otras firmas, es una barrera al ingreso.

El análisis económico de los sistemas agroalimentarios se asocia a estructuras de mercado cada vez menos identificadas con la competencia perfecta. De ahí que el objetivo de este trabajo es el de aproximarse a la definición del mercado de un bien agroalimentario como es el trigo. 


\section{Características del Valle del YaQui: agricultura, tecnología Y COMERCIO}

El valle del Yaqui forma parte de la planicie costera del noroeste de México, en la parte sur del estado de Sonora. Cuenta con una extensión territorial de 2117220 hectáreas, de las cuales 172422 se destinaron a la producción de trigo, principal cultivo de la región, en el ciclo 2010-2011 (Tamayo et al., 2011).

La historia de la producción comercial de este grano en la región data de principios del siglo pasado. Pese al clima desértico del estado de Sonora, el manejo de las cuencas fluviales permite el desarrollo agrícola mediante una inversión en infraestructura (diques, presas y canales) ${ }^{4}$ que garantiza el riego.

Algunos autores, como Hewitt (1978), destacan también el perfil del agricultor sonorense, caracterizado por tener una visión empresarial para la cual especular con la compra y la venta de tierras es un elemento tan importante como la producción y la comercialización, y que ha contado con apoyos de la clase política nacional para la construcción de obra pública o para la obtención de crédito, entre otras cosas.

A la par del desarrollo productivo, se registran avances en la investigación científica regional, como fue el establecimiento del campo experimental El Yaqui en terrenos del valle, en la década de los treinta, y la inauguración, en 1943, de la Oficina de Estudios Especiales (OEE) con el objetivo de propiciar el incremento en la producción de bienes alimenticios; la investigación se realizó fundamentalmente en torno a problemas científicos relacionados con la adaptación de semillas a suelos locales. En la actualidad, el Instituto Nacional de Investigaciones Forestales, Agrícolas y Pecuarias (INIFAP) y el Centro Internacional de Mejoramiento del Maíz y Trigo (CIMMYT) con el apoyo del Patronato para la Investigación Experimental Agrícola del Estado de Sonora (PIEAES), son responsables de la creación de variedades nuevas y de su reproducción.

Aunada a los apoyos con recursos públicos e internacionales, está la experiencia de la iniciativa privada en la generación de variedades agrícolas. Tal es el caso de RSI, empresa conformada por un grupo de productores y un investigador retirado, con el fin de generar una opción para la producción de trigo en el valle del Yaqui. Sin embargo, a más de diez años de su formación, la empresa no

\footnotetext{
${ }^{4}$ El caudal del río Yaqui fluyó libremente hasta 1941, cuando concluyó la construcción de la presa Lázaro Cárdenas (La Angostura). Después fueron construidas la Álvaro Obregón (Oviachic), en 1952, y la Plutarco Elías Calles (El Novillo), en 1965 (Distrito de Riego del Río Yaqui, s.f.).
} 
ha producido variedades que se destaquen o se diferencien de las desarrolladas por el sector público (INIFAP-CIMMYT).

En el valle existen alrededor de 29000 productores, la mayoría registrados como ejidatarios. Sin embargo, a partir de diversas reformas legales en la última década del siglo $\mathrm{xx},{ }^{5}$ cerca de $85 \%$ de ellos rentan su tierra, principalmente a medianos y grandes productores (Almanza, 2008), quienes cultivan trigo y maíz y se asocian en organismos para la comercialización. Actualmente, es posible identificar siete agroempresas trigueras con dichas características (integración de la parte productiva y la comercialización), todas nacionales, aunque una de ellas mantiene vínculos con la transnacional Cargill.

La producción de trigo es vulnerable a dos fenómenos: el clima y los ciclos de la economía internacional. Si bien cuenta con una ventana de oportunidad temprana (en el mundo, la cosecha de trigo cristalino del valle del Yaqui es la primera en salir al mercado), está supeditada a las lluvias que alimentan las presas de la región, las cuales se presentan cada vez con menor frecuencia, lo que a su vez repercute en los costos de producción.

Por otro lado, el precio se fija mediante el contrato de futuros del Chicago Board of Trade, es decir, el trigo cristalino es una mercancía genérica (commodity) cuyo valor depende del uso especulativo que tendrá en el mercado y no de los intereses de comercialización de quien lo produce. Para hacer frente a estos fenómenos, los dividendos obtenidos por la comercialización están sujetos principalmente a los avances científicos que tienen lugar en la región para la obtención de semillas con ventajas que se puedan ver reflejadas en la estructura de costos de producción.

\section{Producción y mercados internacionales de trigo}

La producción mundial de trigo para el año 2012 fue estimada por la Organización de las Naciones Unidas para la Alimentación y la Agricultura (FAO) en 699 millones de toneladas, con una superficie cultivable que no ha tenido cambios e, incluso, muestra una ligera tendencia a la baja (gráfica 1). Este grano se clasifica

${ }^{5}$ El patrón de cultivos en el valle del Yaqui sufrió transformaciones tanto por la reforma de 1992 al artículo 27 de la Constitución Mexicana, como por otros cambios jurídicos de principios de los noventa, como la nueva Ley de Aguas y las modificaciones a la Ley Agraria, de tal manera que el agua pasó a ser propia del distrito de riego, está concesionada a los productores y ellos la administran, además de que la tierra ejidal puede ser rentada y/o vendida legalmente. 
Gráfica 1. Producción y área de cultivo de trigo en el mundo

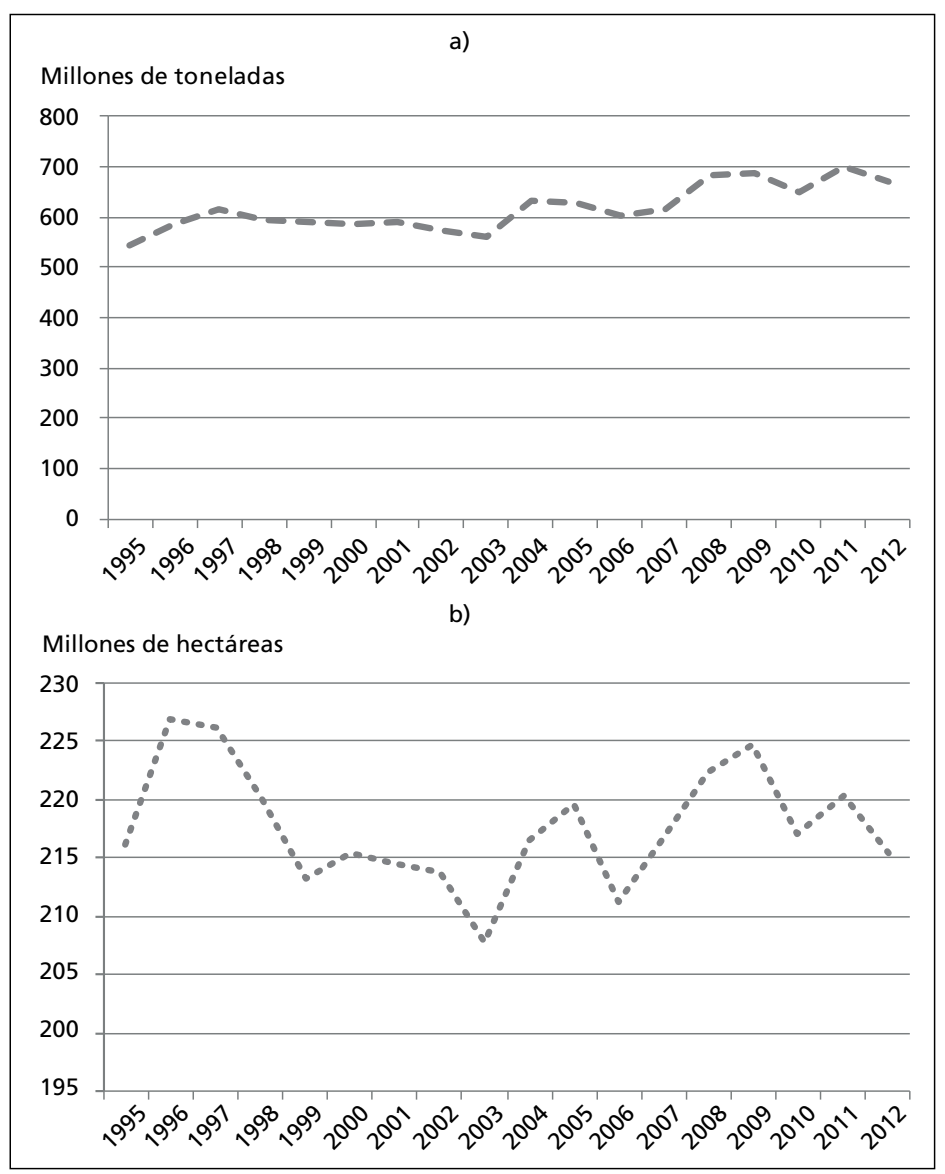

Fuente: Food and Agriculture Organization of the United Nations (2015).

en cinco grupos según las propiedades del gluten: ${ }^{6}$ los primeros tres son trigos panificables y de alta demanda nacional, mientras que el cuarto y el quinto son los denominados duros o cristalinos, destinados a la industria procesadora de pastas y galletas.

La producción mundial de los trigos destinados a pastas durante 2015 fue de 34.1 millones de toneladas, cerca de un millón menos que el ciclo anterior

\footnotetext{
${ }^{6}$ En Estados Unidos y Canadá, los trigos se clasifican por sus hábitos de crecimiento, mientras que en México se clasifican por las propiedades del gluten.
} 
(gráfica 2). Los principales productores son la Unión Europea ${ }^{7}$ y Canadá, que si bien representan un elevado volumen, muestran una caída en los últimos diez años, al tiempo que México y Argelia mantienen una trayectoria ascendente.

\section{Gráfica 2. Evolución de la producción mundial de trigo cristalino} (principales productores)

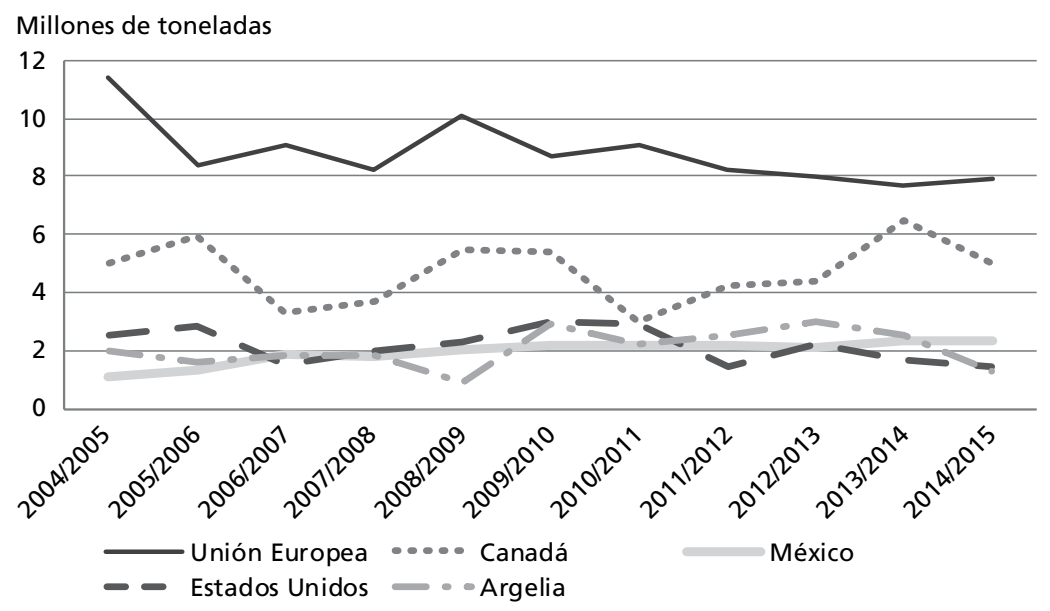

Fuente: FranceAgriMer (2013; 2015).

Respecto al comercio internacional, los principales importadores de 2005 a 2015 fueron la Unión Europea y Argelia (cuya producción mostró un ligero descenso en el último ciclo) ${ }_{,}^{8}$ seguidos de Marruecos y Estados Unidos (panel a) de la gráfica 3), mientras que, como muestra el panel b), el principal país exportador es Canadá, seguido de la Unión Europea y Estados Unidos. México se ubicó como cuarto país exportador durante el ciclo 2012-2015, con 1.1 millones de toneladas. La principal nación consumidora de este grano (ya en forma de pasta) es Italia, con 26 kilogramos por habitante en 2009, de acuerdo con una encuesta de la Unioni Industriali Pastai Italiani (UNIPI). En cambio, según la misma encuesta, en México, cada habitante consume sólo tres kilogramos al año (FranceAgriMer, 2013).

\footnotetext{
${ }^{7}$ En la Unión Europea, el principal país productor es Italia, el cual muestra una trayectoria paralela a la de esta entidad en su conjunto (FranceAgriMer, 2013).

${ }^{8}$ Durante el ciclo 2009-2010, Italia fue el país con mayor volumen de comercio, con una importación de cerca de 21000 toneladas y una exportación de 249000 (FranceAgriMer, 2013).
} 
Gráfica 3. Evolución del comercio la producción mundial de trigo cristalino (principales importadores y exportadores)

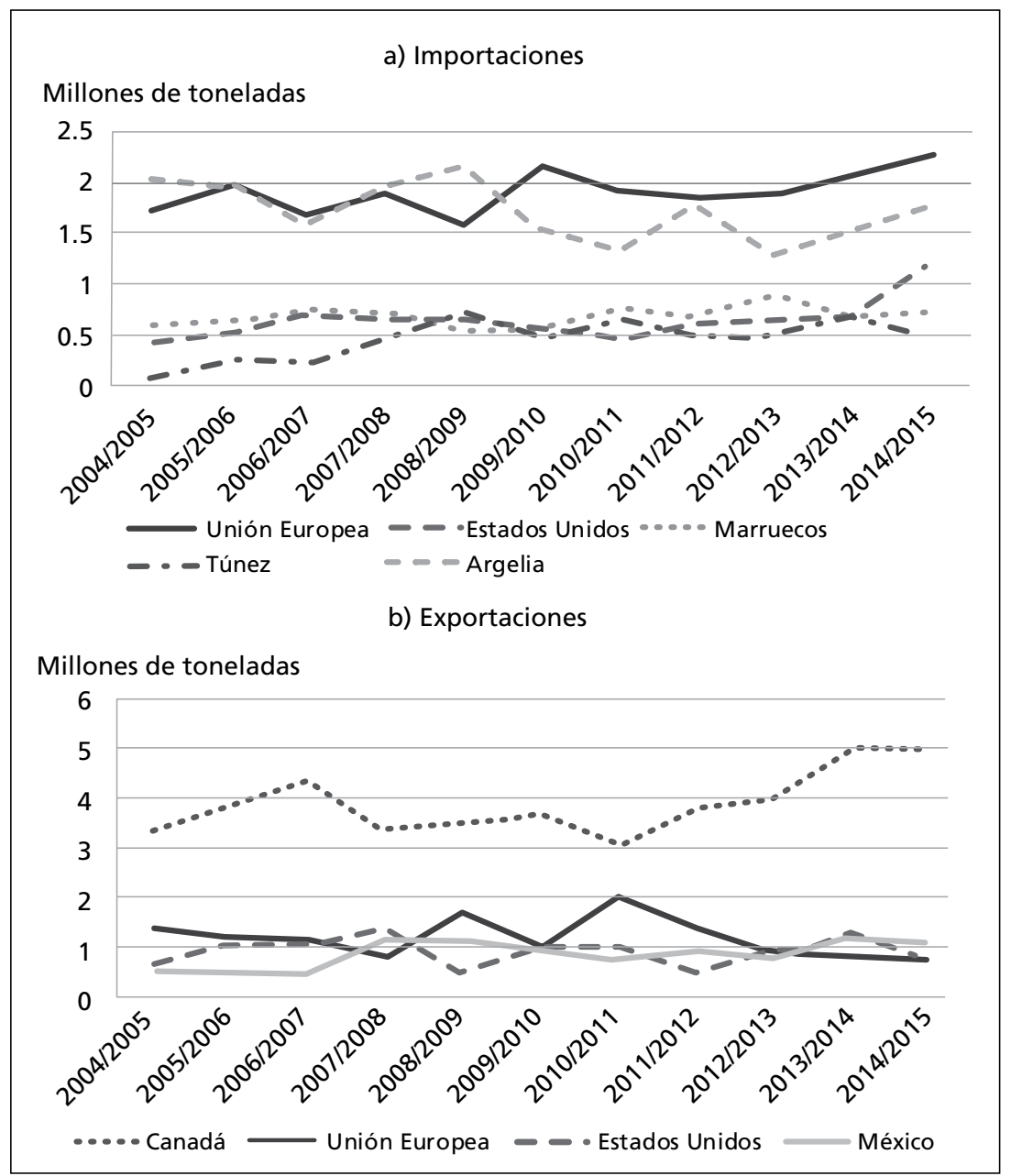

Fuente: FranceAgriMer (2013; 2015).

Los precios internacionales de este grano muestran una relativa estabilidad después de un largo periodo (gráfica 4). En 2008, tuvieron una importante alza, a la que siguió una caída de $30.37 \%$ en 2009 , la más abrupta en veinticuatro años. Sin embargo, hacia 2011 , los precios se recuperaron y crecieron $37 \%$ con respecto al ciclo anterior, lo que pudiera significar una salida a la larga caída de los precios del trigo en el mundo. 
Gráfica 4. Evolución de los precios internacionales de trigo*

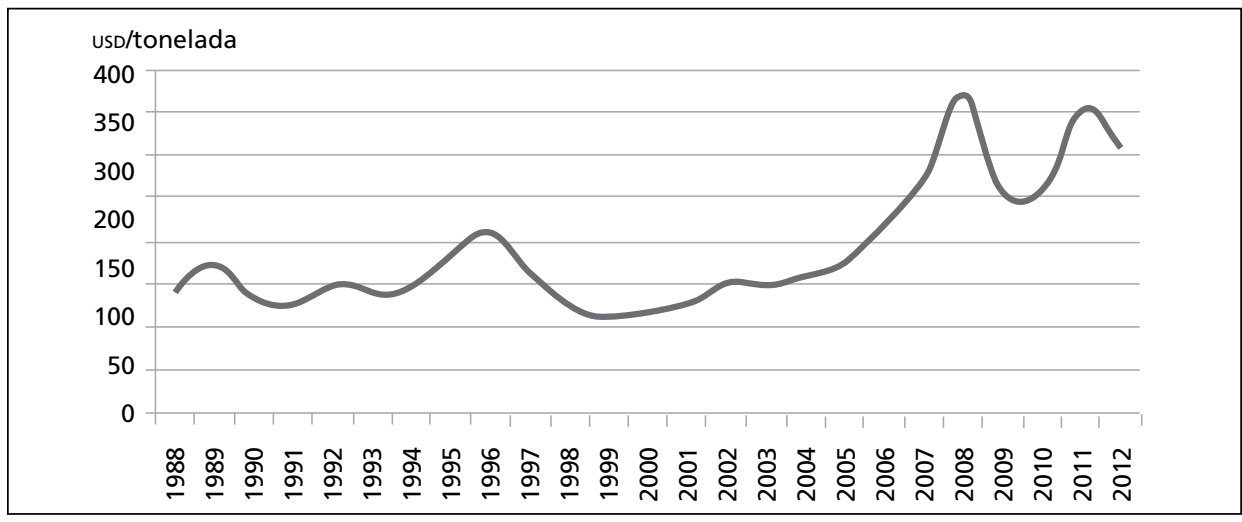

* La cotización de 2012 sólo llega hasta julio.

Fuente: Fedeagro (2015).

\section{Producción y mercados nacionales de trigo}

En México también se siembra trigo panificable, aunque su comportamiento de mercado es diferente al del trigo cristalino, así como sus requerimientos para la producción. Si bien se realiza innovación en las variedades de trigos suaves, su rendimiento y los incentivos a su producción no son suficientes para cubrir la demanda nacional, por lo que en su mayor parte es importado. ${ }^{9}$ Los principales estados productores de trigo son Guanajuato y Sonora. Es en este último donde se obtienen mayores volúmenes de trigo cristalino, que alcanzó durante el ciclo 2010-2012 alrededor de 1.7 millones de toneladas, con un valor de más de cinco mil millones de pesos.

La gráfica 5 confirma lo que se desprende de la sección anterior: las exportaciones de este grano fueron de más del $50 \%$ de la producción. En dicha gráfica pueden observarse dos tendencias: el panel a) muestra que las importaciones de trigo para la industria panificable, particularmente de Estados Unidos, son muy importantes, mientras que el panel b) señala que el consumo pecuario pierde importancia paulatinamente, al tiempo que las exportaciones son cada vez más representativas y la molienda industrial en la producción de pastas y galletas crece ligeramente.

\footnotetext{
${ }^{9}$ En entrevistas, realizadas en octubre de 2012, investigadores del INIFAP señalaron que una de las ventajas de la importación de grano panificable es el transporte vía ferrocarril desde la frontera con Estados Unidos.
} 
Gráfica 5. Evolución de la producción y distribución porcentual del trigo en México

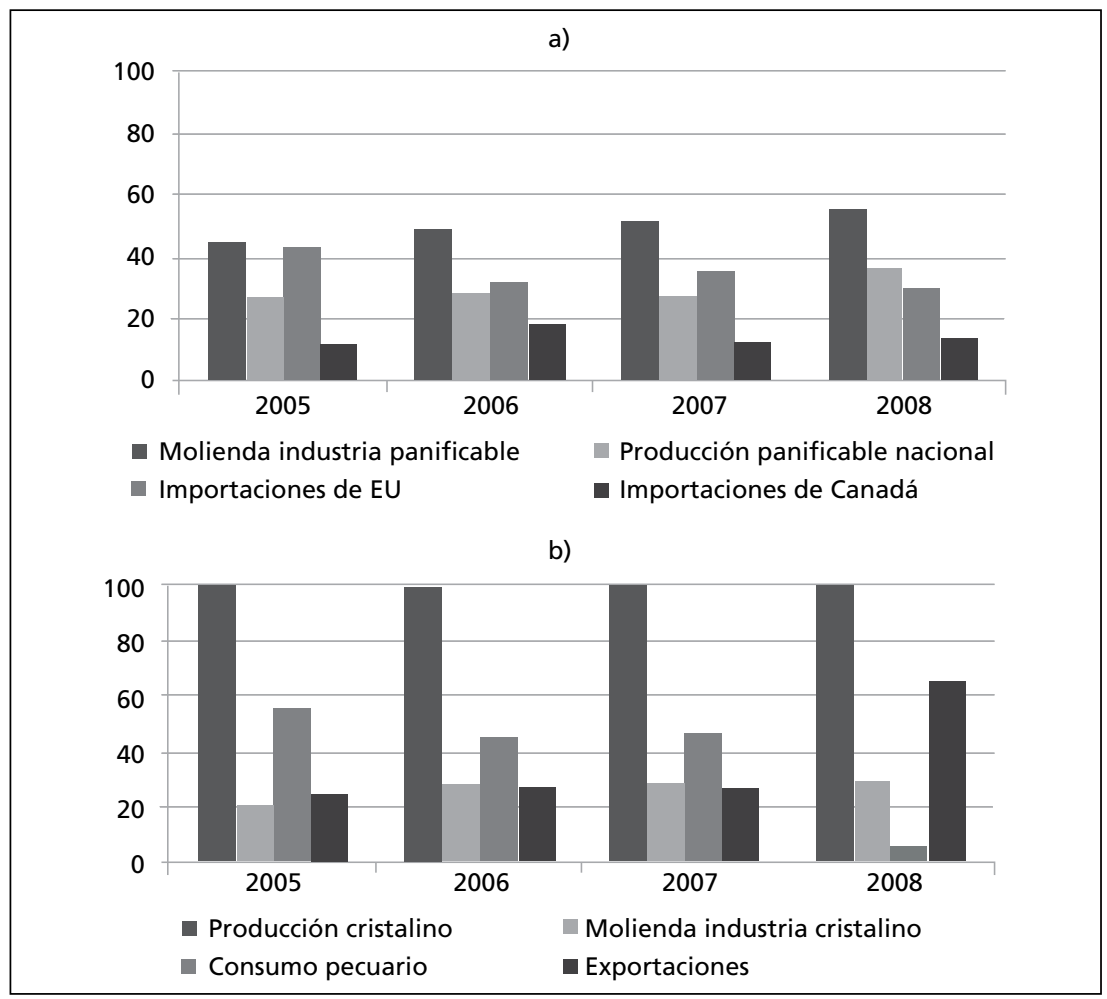

Fuente: Fedeagro (2015).

\section{DIMENSIÓN Y ESTRUCTURA DE MERCADO}

Como se ha señalado, la definición de mercado implica un análisis de su dimensión y de su estructura. Para ello, en este caso, se identificaron los elementos coyunturales del sistema triguero del valle del Yaqui, dando como resultado el diagrama que se presenta en el cuadro 1. Abarca la participación del gobierno como instancia que otorga los apoyos para la producción así como generadora de conocimiento que beneficie al sector; a los organismos de ID (expresada en variedades de trigo mejoradas) tanto del sector público como del sector privado; a las agroempresas identificadas por su capacidad productiva y para la comercialización del grano, y la demanda de este producto, la cual tiene como principal característica que no está representada por un consumidor final, sino por industrias procesadoras. 
Cuadro 1. Sistema triguero del valle del Yaqui

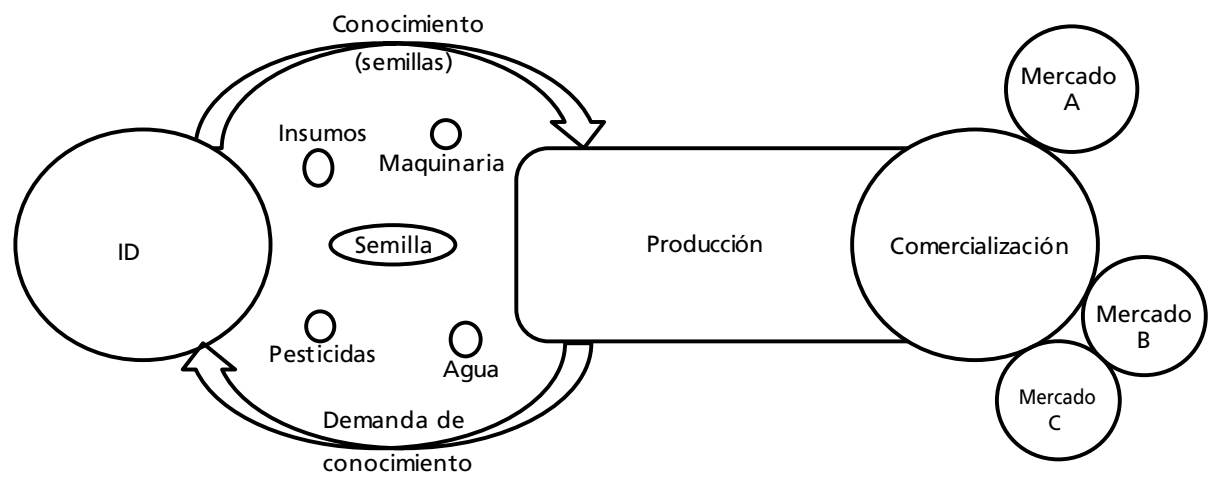

Fuente: Elaboración propia con información obtenida en entrevistas.

De lo anterior, emergen tres preguntas centrales para el análisis: 1) ¿Cuáles son las características del consumidor al que se dirigen las agroempresas del sistema? 2) ¿Qué tipo de producto demanda? 3) ¿Qué condiciones imponen estos consumidores a las agroempresas?

\section{Características del consumidor}

El trigo cristalino que se produce en el valle del Yaqui tiene tres segmentos de venta primordiales: el mercado de alimentos balanceados para el ganado, al que denominaremos mercado pecuario; la industria procesadora de pastas y galletas en México, o mercado industrial, y el mercado de exportación, a países del norte de África y de Sudamérica, y a Italia, principalmente. Como se muestra en la gráfica 5, a diferencia de los trigos suaves, la producción nacional de los cristalinos satisface la demanda interna, de tal manera que no hay volúmenes de importación para el mercado pecuario ni el mercado industrial. Por otro lado, en lo que respecta al mercado de exportación, el valle del Yaqui juega un papel importante con una participación de $42 \%$ del total nacional.

En el cuadro 2 se puede observar los tres tipos de demanda que tiene el trigo cristalino del valle del Yaqui, los cuales están vinculados por tres conceptos principales: mercado, comercialización y calidad. Los dos primeros expresan el destino de la producción, mientras que el tercero contempla las condiciones que debe satisfacer el trigo para ser aceptado en cada uno de los mercados de destino. Conviene advertir que, según los expertos entrevistados, la calidad está asociada 
al nivel de proteína que contienen los granos de trigo o a la pigmentación que presentan, por lo que estos rasgos no son relevantes para el mercado pecuario, ya que demanda principalmente volumen. ${ }^{10}$

\section{Cuadro 2. ¿Quiénes demandan trigo cristalino del valle del Yaqui?}

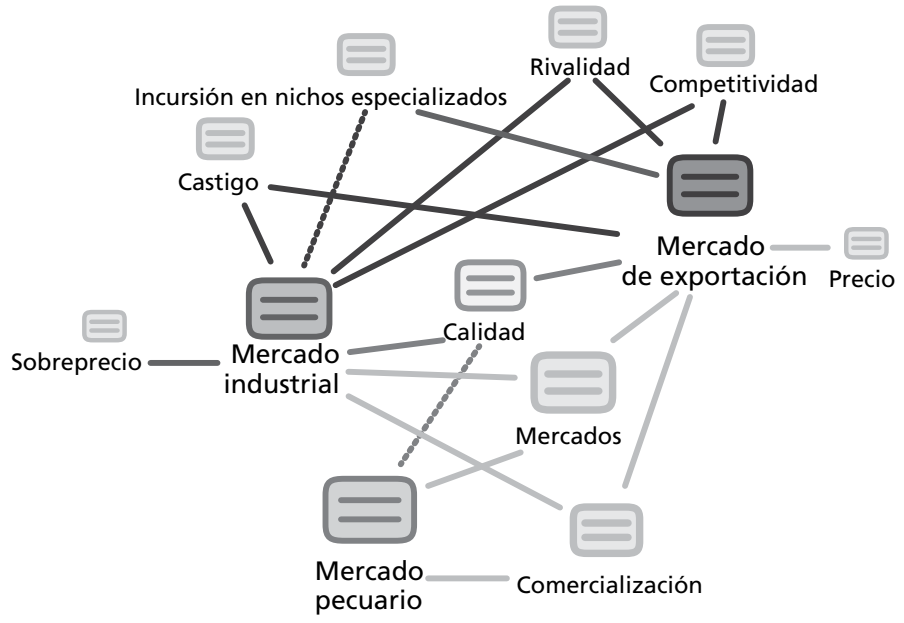

Fuente: Elaboración propia con resultados de entrevistas a expertos.

En segundo orden de importancia están otros conceptos que se asocian sólo al mercado industrial y al mercado de exportación. Al respecto, destaca el código de castigo, ya que según lo señalado por los expertos entrevistados, cuando no se satisfacen los estándares de calidad, el comprador impone alguna penalización, como la disminución de pago por tonelada; por el contrario, el código de sobreprecio se asocia al mercado industrial, en tanto que en éste existe disposición de otorgar un suplemento monetario a quienes cumplan con los niveles de calidad demandados.

Otro de los elementos del cuadro 2 que se asocia a dichos mercados es la incursión en nichos especializados. El resultado del análisis de las entrevistas con expertos, particularmente del mercado de exportación, indica que este código se asocia con el de la calidad, requerida para ser más competitivos al insertarse en nuevos mercados. La ausencia de calidad y la presencia de rivalidad en el

\footnotetext{
${ }^{10}$ Los investigadores estiman una relación inversa entre los niveles de proteína y el volumen de la producción, de tal manera que al obtener una variedad con mayores rendimientos, la proporción de proteína tenderá a disminuir. En la actualidad, en el valle del Yaqui se siembran variedades que pueden generar hasta siete toneladas de trigo cristalino por hectárea.
} 
mercado pecuario dan cuenta de que, a diferencia de los otros dos, éste es un demandante dispuesto a recibir trigo en cualquier condición, lo cual implica que los otros dos mercados se asocian con conceptos como el de sobreprecio, en el caso del mercado industrial, o con el de precio, en el caso del mercado de exportación, es decir, sólo se acude a éste cuando el precio del producto y sus atributos (apoyos y tipo de cambio) son atractivos.

\section{2. ¿Qué tipo de producto se demanda?}

Conocer el tipo de producto que se demanda permite determinar la dimensión del mercado. A lo largo de las entrevistas, los actores fueron indicando atributos comerciales del trigo, vinculados a cada uno de los destinos que éste puede tener, ya sea el mercado pecuario, el industrial o el de exportación.

\section{Cuadro 3. Tipo de producto}

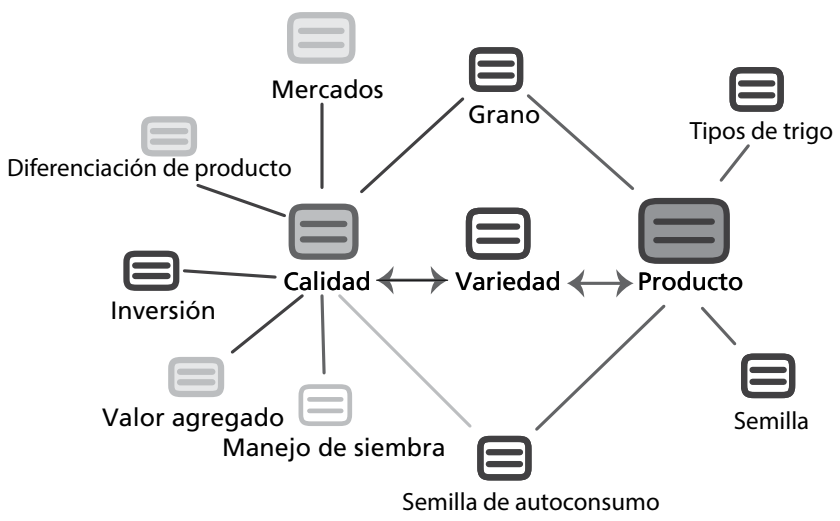

Fuente: Elaboración propia con base en resultados de entrevistas a expertos.

En el cuadro 3 (de derecha a izquierda), se define el tipo de grano a partir de sus características, por ejemplo, la concentración de gluten, en función del mercado de destino. Es decir, si el trigo es harinero, panificable o cristalino; en esta último caso, destinado a los mercados ya descritos. Dado que $97 \%$ de la producción y la comercialización de trigo en la región corresponde al tipo cristalino, la siguiente característica que define al producto se determina al inicio de su producción: si se comercializará como semilla o como grano.

Las variedades de trigo que se producen en el valle del Yaqui garantizan ciertas condiciones de desarrollo de la planta, como resistencia a enfermedades y 
rendimientos elevados. Sin embargo, dadas las características del trigo, el grano puede guardarse, una vez cosechado, para futuras siembras, lo que se conoce como semilla de autoconsumo y que no garantiza los mismos resultados de la semilla original. Por ello, desde el inicio de la producción se determina a qué mercado se acudirá; por ejemplo, para el de exportación, el uso de semilla certificada ${ }^{11}$ garantiza la calidad del grano, a diferencia del pecuario, para el cual puede ser suficiente el uso de semilla de autoconsumo.

\section{Cuadro 4. Relación de la ID con el tipo de producto}

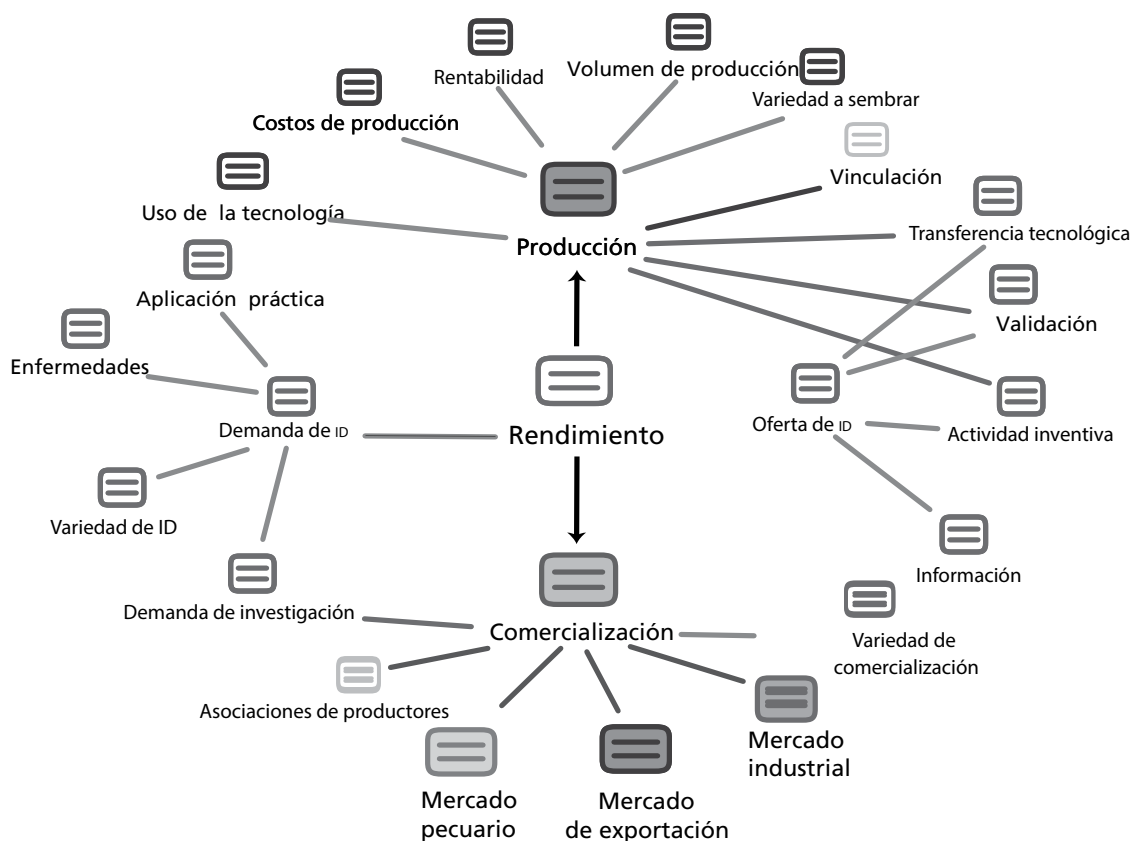

Fuente: Elaboración propia con resultados de entrevistas a expertos.

Las variedades de trigo cultivadas en el valle son reconocidas en el mundo por los volúmenes de producción alcanzables. Dadas las dificultades en los mercados internacionales y la vulnerabilidad del precio, el obtener altos rendimientos es una característica que castiga la calidad del grano sin poner en riesgo

${ }^{11}$ La Secretaría de Agricultura, Ganadería, Desarrollo Rural, Pesca y Alimentación (SAGARPA), a través del Sistema de Inspección y Certificación de Semillas, es la encargada de dar seguimiento a la producción y comercialización de éstas. De esta manera, una semilla certificada garantiza que se obtuvo por métodos que aseguran su calidad genética, física, fisiológica y fitosanitaria. 
su comercialización (cuadro 4), particularmente cuando se tiene un mercado poco exigente, como es el pecuario. Sin embargo, según los expertos entrevistados, un buen manejo de la siembra y de la inversión garantiza que aun estas semillas rendidoras puedan alcanzar niveles de proteína aceptables para el mercado industrial y el de exportación.

En el valle del Yaqui, los organismos generadores de conocimiento (expresado en nuevas variedades de semilla) pertenecen tanto al sector público como a la iniciativa privada. Estos últimos venden las semillas resultantes de su trabajo de investigación, mientras que los primeros transfieren tecnología, además de ofrecer información privilegiada mediante el proceso de validación. Es decir, una vez obtenida una nueva variedad, los investigadores buscan el apoyo de productores con ciertas características (tierra propia y gran número de hectáreas) para verificar la viabilidad de las semillas y, a cambio, reciben información de primera mano sobre su manejo.

De esta forma, el sector productivo puede decidir qué variedad sembrar con base en los criterios de rentabilidad, costos de producción y uso de tecnología, y tomando en cuenta a qué mercado se dirigirá la producción. En consecuencia, sus demandas de ID no sólo tienen que ver con el rendimiento, sino también con la resistencia a enfermedades y con la reducción en el uso de insumos, como el riego, entre otros temas de investigación.

\section{3. ¿Qué condiciones imponen los consumidores a las agroempresas?}

Para conocer la estructura del mercado, es importante analizar las condiciones para el ingreso que cada uno de los mercados de destino impone. En el caso del trigo cristalino del valle del Yaqui, se han identificado tres tipos de mercado, cada uno con sus condiciones particulares. En el cuadro 5 se observan dos esquemas que explican, a través del análisis de la información proporcionada por los expertos entrevistados, la asociación entre códigos y categorías respecto a las exigencias del mercado pecuario y las estrategias que emplean los agroempresarios para cumplir con sus condiciones de ingreso.

El panel a) del cuadro 5 muestra que el mercado pecuario se asocia a códigos como la vinculación, el tipo de trigo y la comercialización. Es decir, ciertos tipos de trigo, cuyas variedades se desarrollan en los organismos y/o empresas ubicados en la región, son relevantes para la comercialización por su volumen de producción (rendimiento). Sin embargo, en las entrevistas se calificaron como irrelevantes para este mercado otros códigos, como la diversificación y la 
calidad. Esta última no fue señalada como una de las exigencias más importantes; asimismo, no mostraron asociación con este mercado el valor agregado, las tendencias, el manejo de siembra y el castigo, lo que evidencia que en este caso simplemente no hay estándares de calidad ni exigencias de ningún tipo.

\section{Cuadro 5. Exigencias y estrategia del mercado pecuario}

a)

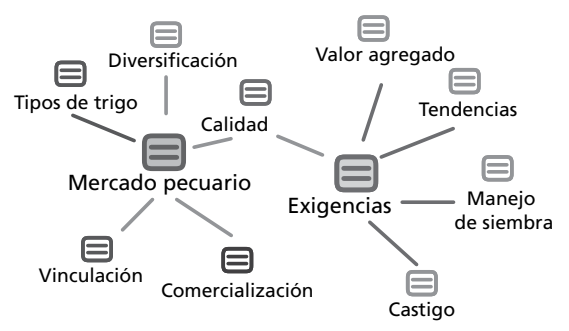

b)

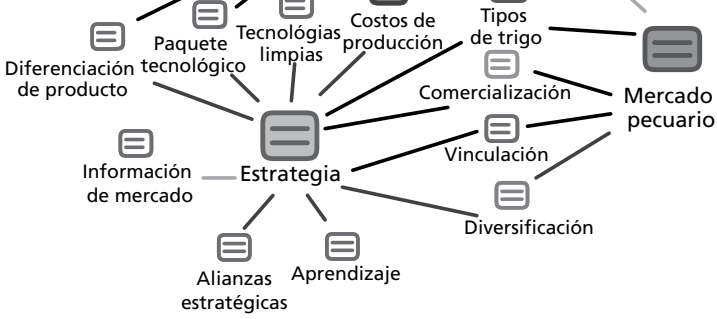

Fuente: Elaboración propia con resultados de entrevistas a expertos.

En cuanto a las estrategias, se hizo una distinción entre aquellas en las que se hace referencia a un comportamiento competitivo y las que siguen una conducta acorde a la definición de estrategia de Martin (1993), quien indica que es toda inversión de recursos que hacen las empresas para limitar las decisiones de sus rivales. En este grupo, entonces, se encuentran códigos como la diversificación, el aprendizaje, la vinculación, las alianzas, la información de mercado. Por su parte, el comportamiento competitivo se asocia a la toma de decisiones, a la planeación y al papel que tienen los derechos de autor (que, en rigor, se denominan derechos de obtentores de variedades vegetales), así como a los atributos de la variedad, principalmente. Ambas categorías se relacionan entre ellas por elementos como los costos de producción, el uso de tecnologías limpias, el paquete tecnológico y la diferenciación.

En el caso del mercado pecuario, los elementos que se asocian a éste igualmente se relacionan con la categoría estrategia, como se observa en el panel b) del cuadro 5, lo que indicaría que los agroempresarios que buscan comercializar su 
producto en este mercado se vincularán con los organismos generadores de conocimiento para alcanzar mayor volumen de producción y para determinar el tipo de trigo a sembrar cada temporada. Están conscientes, además, de que no es relevante la diversificación ni cubrir condiciones de calidad.

\section{Cuadro 6. Exigencias y estrategia del mercado industrial}

a)

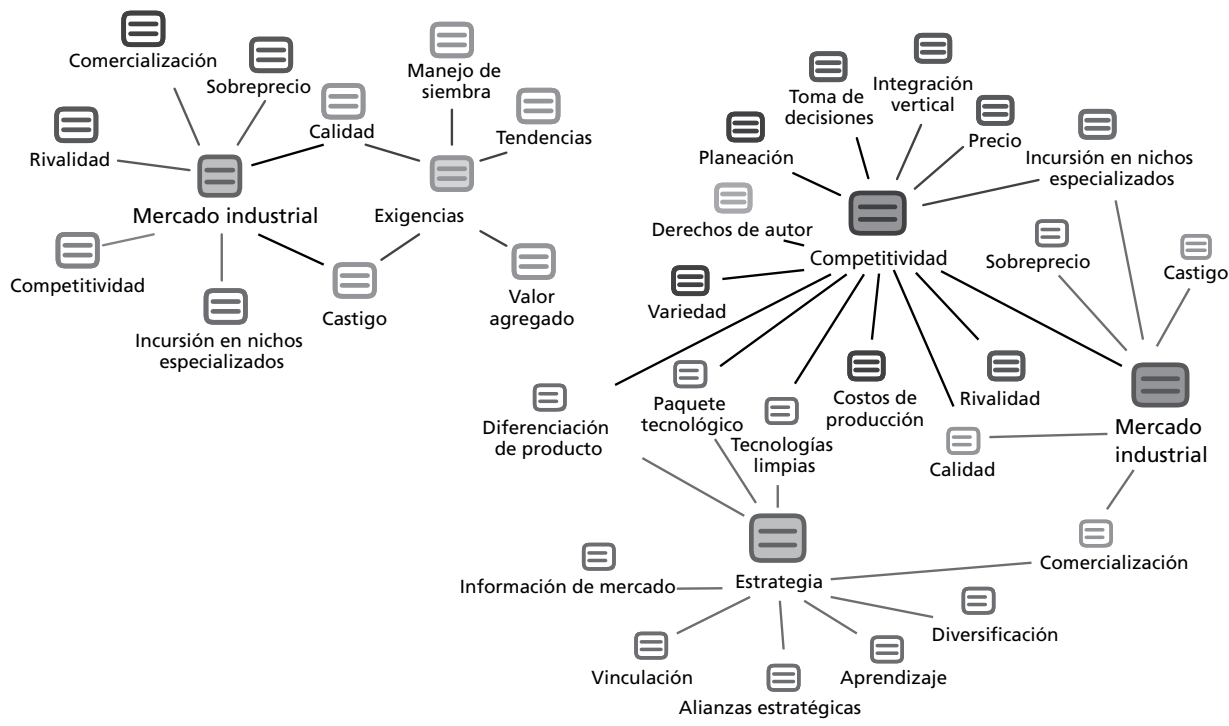

Fuente: Elaboración propia con resultados de entrevistas a expertos.

En cuanto al mercado industrial, la competencia y la rivalidad son conceptos clave para la comercialización en este mercado dispuesto a pagar un sobreprecio a los agroempresarios que provean los porcentajes de calidad que se requieren. Sin embargo, en caso de no cumplir con sus exigencias, se imponen castigos, como se muestra en el panel a) del cuadro 6. La asociación del mercado industrial con la incursión en nichos especializados puede entenderse mediante el concepto de manejo de siembra, ya que es en este sector donde resulta más apremiante que haya un seguimiento de la producción en el campo por parte de expertos, para lograr cumplir las exigencias que impone este mercado, como niveles de nitrógeno y porcentaje de proteína en los granos.

Para que las agroempresas acudan al mercado industrial, deberán, entonces, evitar los castigos y buscar el sobreprecio o premio que ofrecen estos consu- 
midores. Para ello, como muestra el panel b) del cuadro 6, adoptarán estrategias que las lleven a ser más competitivas; en tal sentido, la diferenciación de su producto mediante el uso de tecnologías permitirá el acceso a nichos de mercado especializados y lograr los niveles de calidad convenidos.

Las condiciones para el ingreso al mercado de exportación implican, como ilustra el panel a) del cuadro 7 , cumplir con la ya mencionada calidad y asumir el posible castigo por su ausencia, lo que en este mercado implica grandes pérdidas monetarias. Cabe destacar las múltiples revisiones a las que se enfrenta el producto en su camino a cada uno de los países de destino. ${ }^{12}$

\section{Cuadro 7. Exigencias y estrategia del mercado de exportación}

(a)

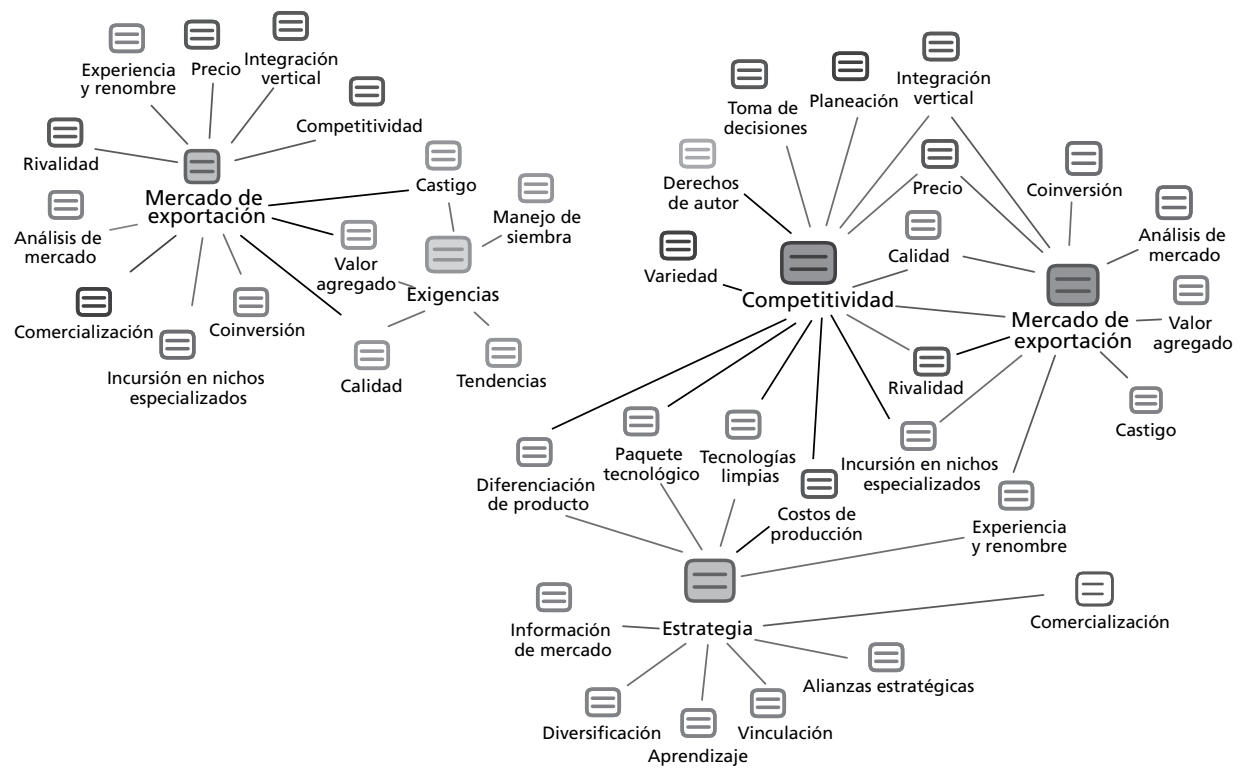

Fuente: Elaboración propia con resultados de entrevistas a expertos.

Aunado a dichos códigos, está el de valor agregado. Según los expertos, la agroempresa exportadora del valle se ve en la necesidad de cuidar su mercado, proveerle de valor y, además, garantizar la certidumbre. Precisamente, su estrate-

${ }^{12}$ El Departamento de Certificación Fitosanitaria de la SAGARPA, de acuerdo con cada país de destino, define los requisitos fitosanitarios para el traslado de trigo. Cuando se detecta grano que no cumple las especificaciones establecidas para México en la Ley Federal de Sanidad Vegetal, se imponen sanciones, lo que evita la propagación de enfermedades y/o plagas en el país y hacia el mundo. 
gia es (como se muestra en el panel b) del cuadro 7) recurrir a su experiencia para ser más competitivos, mediante el uso de tecnologías junto con un constante análisis del mercado y de los precios internacionales. Un elemento importante en la agroempresa que acude al mercado de exportación es la coinversión con una transnacional para fortalecer su compromiso de incursionar en nichos de mercado especializados y resaltar la importancia de conceptos como la experiencia y el renombre. Finalmente, es importante señalar que conceptos como la participación del gobierno y otros que se asocian a la categoría de normatividad, si bien en las entrevistas resultaron de interés, en el análisis no inciden en la definición de mercado.

\section{Conclusiones}

De este análisis se concluye que no se puede hablar sólo de un mercado del trigo cristalino del valle del Yaqui, ya que este grano tiene tres salidas, cada una con características diferenciadas. Esto implica asumir la existencia de otros tantos mercados del trigo cristalino, los cuales enfrentan problemas tales como la volatilidad del precio, sujeto a un tipo de cambio variable, y que enfrentan sus dificultades mediante la ID. Ya sea para obtener mayor volumen, para mantener un estándar de calidad o para solucionar problemas técnicos, cada agroempresa planteará demandas de ID que satisfagan las exigencias que el consumidor les imponga, según el mercado al que se dirijan. Cada uno de estos mercados se define teóricamente por las condiciones y la estructura de mercado, que en este caso se analizó por el tipo de producto y las condiciones para el ingreso. A continuación, se destacan los principales hallazgos de cada definición de mercado.

Mercado pecuario. Básicamente, se trata de la industria procesadora de alimentos balanceados para el mercado porcícola de Sonora. A este mercado acude cualquiera de las agroempresas del valle y su demanda representa cerca de $40 \%$ del total. En este marco, la demanda de trigo se centra en la obtención de grandes volúmenes, sin considerar el porcentaje de proteína del grano o su nivel de humedad, mucho menos la diferenciación del producto. Por lo mismo, no es un mercado exigente y las condiciones que impone para el ingreso prácticamente no existen.

Mercado industrial. Lo constituyen las empresas procesadoras de pastas nacionales y demanda un producto con ciertas características, como un determinado porcentaje de proteína o cierto pigmento, a lo que llaman calidad del grano. Los consumidores están dispuestos a pagar un sobreprecio o premio por ello y, 
sin embargo, la situación económica mundial lleva a los productores a enfocarse en variedades de trigo que en lugar de ser ricas en proteína, tengan altos rendimientos, lo que propicia un incremento de nitrógeno en el grano y una baja de la calidad. Los industriales de este mercado argumentan que con un buen manejo de la cosecha y seguimiento por parte de los investigadores, estas variedades pueden alcanzar los altos rendimientos esperados sin sacrificar el pigmento.

Cumplir con las condiciones para el ingreso a este mercado supone un buen manejo de la producción para alcanzar los porcentajes de proteína que exige. Para ello, cada agroempresa invertirá en tecnología y buscará trabajar en conjunto con expertos, tomando en cuenta el comportamiento de las rivales que acuden a este mercado.

Mercado de exportación. Está constituido principalmente por países del norte de África y de América del Sur; los envíos a esas naciones representan cerca de $35 \%$ de la producción del valle del Yaqui y son realizados principalmente por una agroempresa que trabaja en coinversión con una compañía transnacional. El tipo de producto para este mercado es de calidad debidamente certificada. De esta forma, la agroempresa debe cumplir con la condición de producir un grano a partir de una semilla certificada y con niveles de calidad determinados por el país de destino. Además, se sujeta a las inspecciones y análisis que establece la Ley Federal de Sanidad Vegetal para su almacenamiento y transportación. Otra condición para ingresar a este mercado es invertir en tecnología y desarrollar estrategias a partir de su experiencia y renombre.

Identificar esta segmentación del mercado del trigo producido en la región puede resultar útil para la toma de decisiones tanto de productores como de las instituciones que otorgan los apoyos para la producción, particularmente cuando el sistema se enfrenta a circunstancias que no pueden controlarse, como como son el comportamiento de los precios del trigo y las variaciones climáticas que afectan directamente la producción.

\section{REFERENCIAS BIBLIOGRÁFICAS}

Almanza, María (2008), "Las organizaciones del sector social del Valle del Yaqui”, Frontera Norte, 20 (40), pp. 135-167.

Baker, Jonathan B. (2007), "Market Definition: An Analytical Overview", Antitrust Law Journal, 74 (1), pp. 129-173.

Distrito de Riego del Río Yaqui (s.f.), "Historia", en Distrito de Riego del Río Yaqui. Consultado en mayo de 2013, en: http://drryaqui.org.mx/antecedentes.html. 
Fedeagro (2015), "Precios internacionales de productos agrícolas". Consultado el 19 de junio de 2015, en: http://www.fedeagro.org/preciointer/preciomes.asp.

Food and Agriculture Organization of the United Nations (2015). "Faostat". Consultado el 19 de junio de 2015, en: http://faostat.fao.org.

France Agrimer (2013), Marché du blé dur. Monde, Europe, France. [PDF] Consultado el 19 de junio de 2015, en: http://www.franceagrimer.fr/content/download/ 23517/194926/file/12\%20-\%20Brochure\%20-\%20\%20Etude\%20sur\%20 le\%20march\%C3\%A9\%20du\%20bl\%C3\%A9\%20dur.pdf.

_ (2015), Présentation blé dur. Consultado el 19 de junio de 2015, en: http://www. franceagrimer.fr/content/download/39300/363802/file/06\%20-\%20Pr\%C3 \%A9sentation\%20bl\%C3\%A9\%20dur\%20-\%20juillet\%202015.pdf

Glaser, Barney, y Strauss, Anselm (1999), The Discovery of Grounded Theory: Strategies for Qualitative Research, s.l., Aldine Transaction.

Hewitt, Cinthia (1978), La modernización de la agricultura mexicana 1940-1970, México, Siglo XXI.

Klerkx, Laurence, y Leeuwis, Cees (2008), "Balancing multiple interests: Embedding innovation intermediation in the agricultural knowledge infrastructure", Technovation, 28 (6), pp. 364-378.

Martin, Stephen (1993), Advanced Industrial Economics, Oxford/Cambridge, Blackwell.

Robles, José, y Garza, María Cristina (2011), "Nuevas condicionantes en las organizaciones de los sistemas alimentarios: el caso del sistema vid de mesa de Sonora", en L., Huesca, M. Camberos y C. Calderón (eds.), Bienestar y desarrollo en el siglo XXI, México, CIAD/Plaza y Valdés.

Shepherd, William., y Shepherd, Joanna (2003), The Economics of Industrial Organization, quinta edición, Long Grove, Waveland Press.

Smits, Ruud (2002), "Innovation studies in the 21st century: Questions from a user's perspective", Technological Forecasting and Social Change, 69 (9), pp. 861-883.

Suárez, Ruth, y Bejarano, Edgar (2001), "Modelos de organización de empresas agropecuarias", Documento de Trabajo 9, CEGA y Conciencias.

Taddei, Cristina (2008), Estrategias de mercado en la industria alimentaria. Plantas del noroeste de México, México, CIAD/Plaza y Valdez.

Tamayo, Luis M.; Ortiz, José E.; Armenta, Inés; Félix, Pedro; Cortés, Juan M.; Ortiz, Alma A.; Fuentes, Guillermo, y Cortez, Edgardo (2011), Agronomía del trigo en el sur de Sonora, Ciudad Obregón, Instituto Nacional de Investigaciones Forestales, Agrícolas y Pecuarias. 